\begin{tabular}{llllr} 
Volumen 34(1):27-35 & (cc) EY-No & ISSN Impreso 0120-0135 & e-ISSN 2256-2273 & doi: http://dx.doi.org/10.22267/rcia.183502.89 \\
\cline { 2 - 6 }
\end{tabular}

\title{
Efecto de la aplicación de copolímeros sobre el cultivo de lechuga (Lactuca sativa $\mathrm{L}$.)
}

\author{
Effect of the application of copolymere on lettuce (Lactuca sativa L.) crop
}

\author{
Horacio Andrada1; Gabriela Di Barbaro²
}

1 Ingeniero Agrónomo. Universidad Nacional de Catamarca. Argentina, horacio_andrada@yahoo.com.ar.

2 Especialista en Metodologías de la Investigación Científica. Universidad Nacional de Catamarca. Argentina, gabydibarbaro@ yahoo.com.ar.

Citar: Andrada, H. \& Di Barbaro, G. (2018). Efecto de la aplicación de copolímeros sobre el cultivo de lechuga (Lactuca sativa L.). Revista de Ciencias Agrícolas. 35(2): 27-35. doi:http://dx.doi.org/10.22267/ rcia.183502.89

Recibido: septiembre 06 de $2016 . \quad$ Aceptado: febrero 27 de 2018.

\section{RESUMEN}

La problemática de la producción agrícola en las regiones áridas y semiáridas reside mayormente en la escasez de agua para riego y en la baja calidad de los suelos, por lo cual, es importante buscar alternativas y formas eficientes de manejo de los recursos hídricos en estas regiones en un marco de absoluta racionalidad y sustentabilidad. El objetivo de este trabajo fue evaluar la capacidad de retención hídrica del copolímero de acrilamida y acrilato de potasio, como mejorador de la implantación y crecimiento de un cultivo de lechuga (Lactuca sativa L.) bajo condiciones controladas. Se compararon dos tratamientos con dosis de 25 y $50 \mathrm{~kg}^{\text {. ha- }}{ }^{-1}$ versus un testigo de control, con el fin de determinar si el uso de dicha enmienda mejoraba la producción del cultivo. Se evalúa con la emergencia, la supervivencia, el peso fresco y seco de la parte aérea del cultivo y el contenido de humedad del suelo. Las respuestas fueron positivas para la aplicación de la enmienda en la dosis menor dado que aumentó la emergencia de plántulas, supervivencia, crecimiento y en consecuencia su rendimiento, como así también el contenido de humedad del suelo. En conclusión, la aplicación de $25 \mathrm{~kg}^{\text {. ha-1 }}{ }^{-1}$ del copolímero en el establecimiento del cultivo de lechuga, mejoró el crecimiento e incrementó su rendimiento.

Palabras clave: acrilamida, acrilato de potasio, hidrogel, rendimiento. 


\begin{abstract}
The main problem of the agricultural production in arid and semiarid regions is mainly the scarcity of irrigation water and the low quality of the soils. Hence, it is important to find alternatives and efficient water resources management practices in these regions within a frame of absolute rationality and sustainability. The objective of this work was to assess the water retention capacity of acrilamyde copolymer and potassium acrylate and how these could improve a lettuce crop (Lactuca sativa L.) implantation and growth under controlled conditions. Two treatments with 25 and $50 \mathrm{~kg}$. ha- $\mathrm{doses}^{-1}$ versus a control sample were compared in order to determine if the use of said amendment could improve crop production. The emergence, survival, fresh and dry weight of the aerial part of the crop and the soil humidity levels were evaluated. The answers were positive for the application of the amendment in the lower dose since the emergence of seedlings, its survival, its growth and its consequent yield raised, as well as the moisture content of the soil. In conclusion, the application of $25 \mathrm{~kg} \mathrm{ha}^{-1}$ of the copolymer in the of the lettuce crop site, improved the growth and increased its yield.
\end{abstract}

Key words: Acrilamyde, potassium acrylate, Hydrogel, yield.

\section{INTRODUCCIÓN}

El agua ha sido considerada desde siempre como el factor que más incide en la producción de alimentos en el mundo. El estrés hídrico es a menudo el factor ambiental más importante que afecta la sobrevivencia, el crecimiento y el desarrollo de las especies vegetales.

Una problemática seria de la producción agrícola de las regiones áridas y semiáridas es la escasez de agua, por tanto, es importante buscar alternativas y formas de manejo de los recursos hídricos de la región para una aplicación racional y sustentable del agua. Como una solución a este problema se propone la utilización de una enmienda de polímeros, pero debido a la escasa información científica se hace necesario estudiar el efecto de este tipo de producto sobre la producción agrícola para dar una respuesta al medio sobre la eficiencia de esta alternativa de producción. Se plantea el interrogante si esa agua absorbida estaría disponible para las raíces de los cultivos y si éste tipo de producto tiene la cualidad de almacenar agua que sea de fácil disponibilidad para los cultivos.

Conocer el estado hídrico de las plantas es importante en muchas situaciones de cultivo, y una de las variables que describen el estado hídrico es el contenido hídrico relativo al peso seco: que está dado por la diferencia entre peso fresco y peso seco dividida por el peso seco (García y Fuentes, 1994).

Los retenedores de agua, hidro-retenedores, hidrogeles ó polímeros superabsorbentes pueden ser definidos como materiales constituidos por redes poliméricas hidrofilias química y físicamente reticuladas, capaces de absorber 0 retener grandes cantidades de agua o fluidos biológicos sin perder su forma tridimensional (3D). Son fácilmente sintetizados por copolimerización de solución de acrilamida y acrilato con un agente reticulante (Brito et al., 2013; Magalhães et al., 2013). El copolímero de acrilamida y acrilato de potasio ó poliacrilato tienen la característica de ser altamente higroscópicos; es decir, absorben grandes cantidades de humedad (finalmente agua). Su presentación, en gránulos o polvo, puede absorber hasta 500 veces su peso en agua (Trujillo, 2009).

Dicho de otra manera, ocho gramos de polvo, serían capaces de retener hasta 4 litros de agua. La vida útil máxima es de hasta 10 años, donde se disminuye su capacidad de absorción alrededor del 10\% al año, durante los cuales se provee de un suplemento regular de agua y así se evita el 
estrés hídrico, se fomenta la aireación, se evita la percolación y la evaporación. Las partículas de acrilato de potasio pueden secarse y volver a hidratarse cada ciclo de cultivo (Trujillo, 2009). Sin embargo, tanto la vida útil del compuesto, como la capacidad de absorción varían según distintos investigadores y se estima que dichas diferencias se deben a la calidad del producto comercial.

El polvo granulado de acrilato de potasio es biodegradable no tóxico que comercialmente se conoce como silos de agua, el cual es un compuesto de copolímeros reticulados de ácido acrílico y acrilamida con base en potasio no tóxico, ni contaminante para el suelo o plantas (Trujillo, 2009). Son utilizados en la actividad agrícola como acondicionadores de suelo, principalmente por aumentar la capacidad de almacenar agua en el suelo (Brito et al., 2013; Magalhães et al., 2013). Los suelos que reciben poca lluvia o irrigación o son demasiado porosos para retener la humedad en la zona de las raíces, se benefician notablemente con el uso de hidrogeles. Adicionalmente, en suelos arcillosos los hidrogeles ayudan a mejorar el contenido de aire del suelo dado el proceso de expansión que se genera por los ciclos de absorción-liberación del material (Barón et al., 2007).

Nissen y Ovando (1999) evaluaron el efecto de un hidrogel humectado aplicado a las raíces de Nothofagus obliqua y Nothofagus dombeyi durante el trasplante y concluyeron que en los tratamientos con hidrogel en Nothofagus dombeyi se obtuvo mayores valores de supervivencia que en aquellos en los que no se utilizó el producto. Observaron que al no utilizar el hidrogel, se obtiene un porcentaje significativamente menor de sobrevivencia $(66,7 \%)$ en la plantación.

En Colombia, Barón et al. (2007) estudiaron el uso de hidrogeles y su efecto en las propiedades hidráulicas de suelos arcillosos para sistemas de hortalizas y establecieron su utilización como una alternativa válida para la conservación de agua en el suelo, mejorando sus propiedades de retención y liberación. Los retenedores de agua son convenientes en condiciones donde es importante la velocidad de retención de agua y el poco tiempo de acceso a fuentes de agua.

En Brasil, se han aplicado hidrogeles a los suelos en los que se cultivan café, frutas cítricas, legumbres y verduras. También se estudió el efecto de los hidrogeles en la siembra y producción de plantines de eucalipto (Lópes et al., 2010) y café, en la supervivencia de Pinus y Araucaria, y en la producción de caña de azúcar (Magalhães et al., 2013). Los hidrogeles se emplean también en campos deportivos y jardines para disminuir los costos de mantenimiento y conservar la frondosidad (Barón et al., 2007). También, se aplican estos materiales en productos de higiene personal, liberación controlada de fármacos, materiales de construcción, cableado eléctrico y sensores entre otras aplicaciones (Barón et al., 2007).

El objetivo principal de esta investigación fue evaluar el uso del copolímero de acrilamida y acrilato de potasio aumenta en la disponibilidad del agua útil para la planta para promover su supervivencia y crecimiento de plantas de lechuga (Lactuca sativa L.) establecidas en cámara de cultivo.

\section{MATERIALES Y MÉTODOS}

Se realizaron dos ensayos en cámara de cultivo, con temperatura, riego e iluminación controlada. En cada ensayo se utilizaron contenedores rectangulares de $25,5 \mathrm{~cm}$ de largo, $14 \mathrm{~cm}$ de ancho y $12 \mathrm{~cm}$ de profundidad con bandeja colectora de drenaje, a los cuales se les agregó $4 \mathrm{~kg}$ de suelo franco arenoso y se cultivó lechuga (Lactuca sativa L.) variedad Walldman Green, con un porcentaje de germinación del 85\% (ISTA, 2014).

El diseño experimental utilizado fue el de Completamente al Azar, con tres tratamientos: T0, T1 y T2 (Tabla 1), diez repeticiones de cada tratamiento y la unidad experimental constituida por 
cada contenedor, para cada experimento. El factor principal de análisis fue la dosis del copolímero de acrilamida y acrilato de potasio.

Tabla 1. Tratamientos establecidos en los ensayos.

\section{Tratamientos}

T0 Testigo (sin adición del copolímero)

T1 Aplicación de $50 \mathrm{Kg}_{\text {.ha-1 }}{ }^{-1}$ de copolímero

T2 Aplicación de $25 \mathrm{Kg}_{\text {.ha-1 }}{ }^{-1}$ de copolímero

Se pesó el copolímero con balanza analítica, se lo incorporó al suelo y se mezcló perfectamente. Luego se aplicó un riego a saturación (1 litro de agua por contenedor en el día). Este volumen de saturación fue determinado mediante pruebas previas. Los contenedores se colocaron en cámara de cultivo con condiciones óptimas para el desarrollo del cultivo $\left(32 \pm 2^{\circ} \mathrm{C}\right.$, fotoperiodo de 16 horas de luz y 8 h de oscuridad y $35 \pm 2 \%$ de humedad). A la semana, con el suelo a capacidad de campo, se sembraron 100 semillas de lechuga por contenedor.

Se realizaron conteos periódicos de semillas emergidas y plántulas. Al finalizar el ensayo, se evaluaron peso fresco y peso seco de parte aérea y contenido de humedad del suelo.

La determinación de la humedad del suelo se realizó por el método gravimétrico, que consiste en el pesado y secado en estufa de la muestra de suelo, donde la humedad de un suelo es la relación expresada en porcentaje entre el peso del agua existente en una determinada masa de suelo y el peso de las partículas sólidas (ISO 11465, 1993; González, 2009).

El efecto de los factores de estudio sobre el crecimiento de las plantas se determinó mediante el Análisis de Varianza y la prueba de comparación de medias mediante Tukey $(\alpha=0,05$ y 0,01$)$, utilizando el software estadístico InfoStat versión 2008 (Di Rienzo et al., 2008).

\section{RESULTADOS Y DISCUSIÓN}

Emergencia de plantas de lechuga. A los cinco días de realizada la siembra se observó la aparición de las primeras plántulas de lechuga sobre la superficie del suelo y a los 14 días de la siembra con las dos hojas cotiledonales extendidas, momento en que se cuantificó emergencia. El análisis estadístico de los datos de plántulas emergidas determinó que no existen diferencias significativas entre los tratamientos. Estos resultados similares se deben al alto contenido de humedad del suelo de todos los contenedores al iniciar el experimento, el cual es necesario para inducir a la germinación de las semillas de lechuga.

Supervivencia de plantas de lechuga. El análisis estadístico de los datos de supervivencia de las plantas de lechuga, 60 días después de la siembra, reveló diferencias significativas entre los tratamientos. Los mejores resultados se obtuvieron con el tratamiento $\mathrm{T} 2$, con el suelo acondicionado con el copolímero de acrilamida y acrilato de potasio con la dosis de $25 \mathrm{~kg}$. ha ${ }^{-1}$, mientras que en los tratamientos restantes (T0 y T1) se obtuvieron similares resultados entre ellos, sólo registrándose diferencias significativas con el T2. El porcentaje de plantas de lechuga que prosperaron fue del 50\%. Mientras que en el tratamiento T1 (50 kg. ha ${ }^{-1}$ ) sobrevivieron el 35\% de las plántulas y las del tratamiento testigo prosperaron alrededor del 25\% de la población de plántulas emergidas (Figura 1). Estudios realizados por Dranski et al. (2013) con Jatropha curcas, indicaron que la inmersión de la raíz en hidrogel, incrementó el porcentaje de supervivencia de las plántulas en un 6,7\%. Pedroza-Sandoval et al. (2015), determinaron que la aplicación del hidrogel en dosis de 12.5 y $25 \mathrm{~kg} \mathrm{ha}^{-1}$ beneficia el crecimiento y productividad del maíz, al aumentar en promedio 31.5 \% el contenido de humedad edáfica, y estimaron que este aumento en la humedad disponible para la planta inducido por el hidrogel, no influyó en la germinación de semillas, ni en la emergencia de plántulas. 


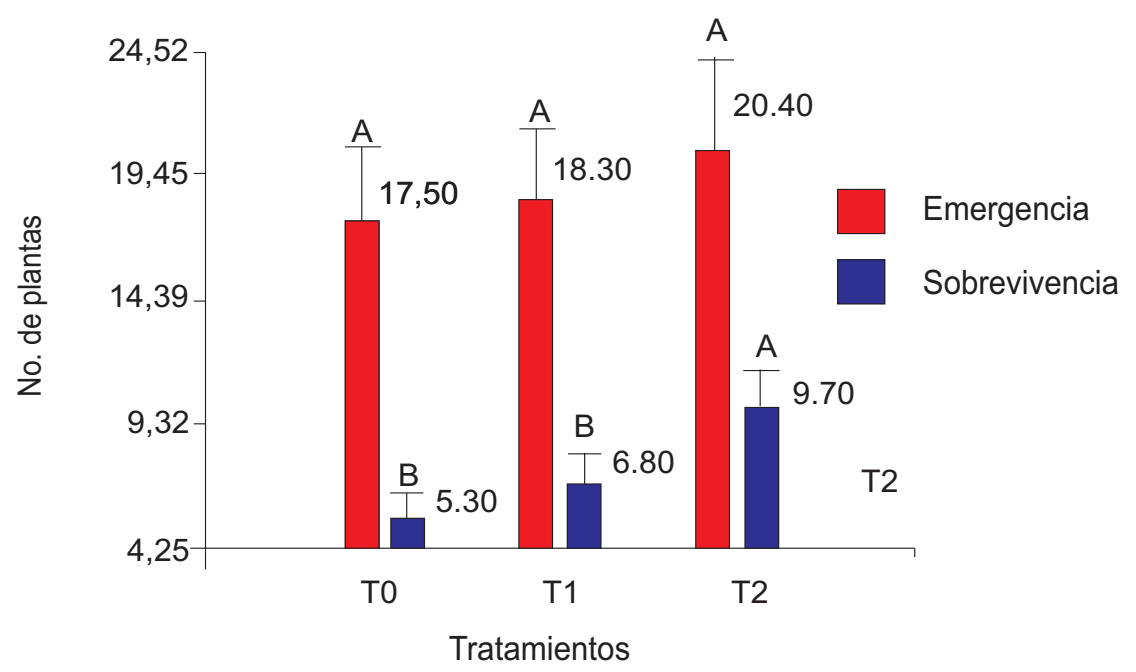

Figura 1. Emergencia y sobrevivencia (\%) promedio de plántulas de lechuga con diferentes dosis de enmienda de copolímero de acrilamida y acrilato de potasio.

Se estima que el mayor contenido de humedad del suelo logrado con la dosis más alta de la enmienda orgánica ensayada (T1), afectó las condiciones de sanidad del cultivo, aumentó la mortandad de plántulas y disminuyó la cantidad de plantas de lechuga que prosperaron hasta el final de la experiencia.

Además, las plantas testigo no lograron superar las condiciones de sequedad del suelo y llegaron al punto de marchitez permanente, lo cual aumentó la mortalidad de plantas por no tener la humedad necesaria para sobrevivir. La enmienda del copolímero retrasó los síntomas de déficit hídrico influyendo en la sobrevivencia. Similar conclusión obtuvieron Lópes et al. (2010) utilizando hidrogel en la producción de plantas de Eucalyptus sp. Sin embargo, Barreto (2011) concluyó que el uso de retenedores de agua y riegos de apoyo, estadísticamente no presentaron diferencias significativas en la sobrevivencia durante el establecimiento de plantaciones forestales de Juniperus flaccida, durante el primer año en campo.

Peso fresco de parte aérea. El Análisis de la Varianza del peso fresco de la parte aérea de plantas de lechuga determinó diferencias significativas entre los tratamientos T2 y T0, donde los mejores resultados se obtuvieron con el tratamiento $\mathrm{T} 2$ $(23,71)$. Sin embargo, no se registraron diferencias estadísticamente significativas entre los tratamientos T1 $(21,67)$ y T2 $(23,71)$, ni tampoco entre T1 $(21,67)$ y T0 $(15,46)$, donde los valores más bajos de peso fresco correspondieron al tratamiento testigo T0 $(15,46)$ (Figura 2).

El mejor rendimiento del cultivo se obtuvo con la

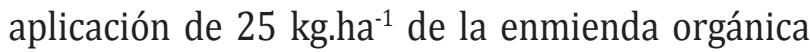
al suelo, con un $53,4 \%$ por encima al tratamiento testigo, mientras se obtuvo un incremento del $40,2 \%$ respecto al testigo con la aplicación de 50 kg.ha-1 de la enmienda (Figura 2).

El mayor crecimiento de plantas de lechuga se logró con la aplicación del hidrogel de acrilamida y acrilato de potasio, resultados coincidentes con el trabajo de Chatzopoulos et al. (2000) en el cual determinaron que en todos los suelos experimentados acondicionados con hidrogel se observó mayor crecimiento del cultivo de rábanos que en el suelo testigo.

Estudios realizados en Brasil, con caña de azúcar determinaron que la aplicación del polímero como hidrogel aumenta el macollaje, lo que proporciona una mayor productividad. Incluso observaron que 
las plantas tratadas con el hidrogel en el segundo ciclo de cultivo, tenían mejores rendimientos (Marques et al., 2013).

Por otra parte, el efecto del hidrogel sobre el crecimiento de las plantas está influenciado por las condiciones agroclimáticas ocurrentes durante el ciclo del cultivo, resultando más efectivo cuando las condiciones son secas. Dranski et al. (2013) observaron que el uso del hidrogel no propiciaba un aumento significativo del crecimiento de plantas de Jatropha curcas L., especialmente para la temporada de siembra de invierno, pero si un aumento mayor que el crecimiento de las plantas sembradas en primavera.

Peso seco de parte aérea y contenido hídrico de plantas de lechuga. El Análisis de Varianza de los valores del peso seco de la parte aérea de plantas de lechuga determinó diferencias significativas entre los tratamientos. Los mejores resultados, que se muestran en la Figura 2, se obtuvieron con el tratamiento T1 (dosis de 50 kg.ha-1). Entre los tratamientos $\mathrm{T} 1$ y $\mathrm{T} 2$, los resultados fueron similares sin registrarse diferencias estadísticamente significativas. Situación parecida se observó entre el testigo y el tratamiento T2 (dosis de $25 \mathrm{~kg} \mathrm{ha}^{-1}$ ), dado que no se registraron diferencias estadísticamente significativas entre el peso seco promedio de los tratamientos, donde los valores más bajos correspondieron al tratamiento testigo (T0).

Con la aplicación de 50 kg.ha-1 de la enmienda orgánica del copolímero de acrilamida y acrilato de potasio al suelo se obtuvieron los valores más altos de peso seco, siendo el incremento del 74\% por encima del testigo, mientras que con la aplicación de $25 \mathrm{~kg}$.ha ${ }^{-1}$ de la enmienda se obtuvo un $38 \%$ más de peso seco que el testigo (Figura 2). Los resultados de peso seco de la parte aérea indican que las plantas del tratamiento T1 presentan un mayor contenido de sustancias orgánicas sintetizadas por las plantas durante el proceso fotosintético y sales minerales acumuladas. Este tratamiento permite una mayor disponibilidad de nutrientes minerales, que entran a la planta en general en forma de iones inorgánicos disueltos en el agua que absorben las raíces (Killian, 2010).

El análisis estadístico de los datos del contenido hídrico de plantas de lechuga estableció similitudes entre los tratamientos, sin registrarse diferencias estadísticamente significativas. Esto puede explicarse, porque en el momento de realizar la extracción y las mediciones de las plantas, estaban turgentes y no se detectó marchitamiento ni síntomas visibles de déficit hídrico.

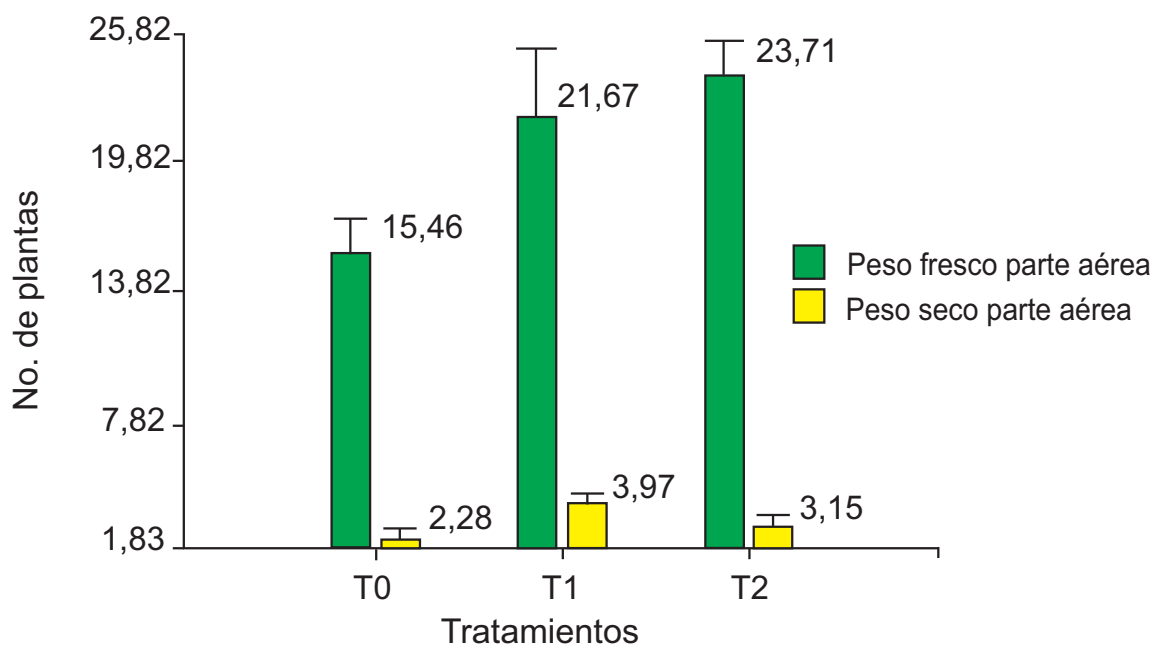

Figura 2. Peso fresco y seco promedio (gramos) de la parte aérea de plantas de lechuga, de tres tratamientos con diferentes dosis de enmienda de copolímero de acrilamida y acrilato de potasio. 
Humedad del suelo. El Análisis de la Varianza del contenido de humedad del suelo en el momento de la cosecha establece diferencias altamente significativas entre los tratamientos para este factor de estudio. El mayor contenido de humedad (del $19,2 \%$ ) se encontró en el tratamiento T1 con la aplicación de $50 \mathrm{~kg} \cdot \mathrm{ha}^{-1}$ del copolímero retenedor de agua y resultados similares (18,6\%) se obtuvo en el tratamiento T2 con la dosis de $25 \mathrm{~kg} \mathrm{ha}^{-1}$ sin ser estas diferencias estadísticamente significativas (Figura 3). Sin embargo, se registraron diferencias altamente significativas con el tratamiento testigo. Los incrementos del contenido de humedad de los suelos con la aplicación de la enmienda de copolímero de acrilamida y acrilato de potasio con respecto al testigo fueron 13,6 y 9,6\% para los tratamientos $\mathrm{T} 1$ y T2, respectivamente. Estos resultados muestran que la enmienda del copolímero de acrilamida y acrilato de potasio mejoró la retención de humedad del suelo lo cual coincide con lo observado por Trujillo (2009).

Además, Irurtia et al. (2012) al estudiar el efecto de polímeros de poliacrilamida en las propiedades físicas de un suelo arenoso, concluyeron que dicho compuesto produce cambios importantes en las propiedades hidrofísicas del suelo, debido a que aumentan notablemente la capacidad de retención del agua y disminuye su conductividad hidráulica. Por otra parte, la capacidad de hinchamiento del polímero de poliacrilamida tiende a descompactar el suelo cuando está húmedo. Los suelos arenosos caracterizados por su alta tasa de infiltración y baja retención de agua pueden mejorar la eficiencia del uso del agua de riego.

En investigaciones realizadas por Chatzopoulos et al. (2000) se informó sobre la diferencia de humedad de los suelos acondicionados con hidrogeles con respecto al testigo. Para un mismo régimen de irrigación los suelos acondicionados con los hidrogeles presentaban mayor contenido de humedad en relación al testigo. Al restringir el riego y someter a las plantas a condiciones de marchitez, las plantas no murieron con la misma velocidad que en el suelo testigo, de ahí que los investigadores infieren un proceso de liberación controlada del agua por parte del hidrogel.

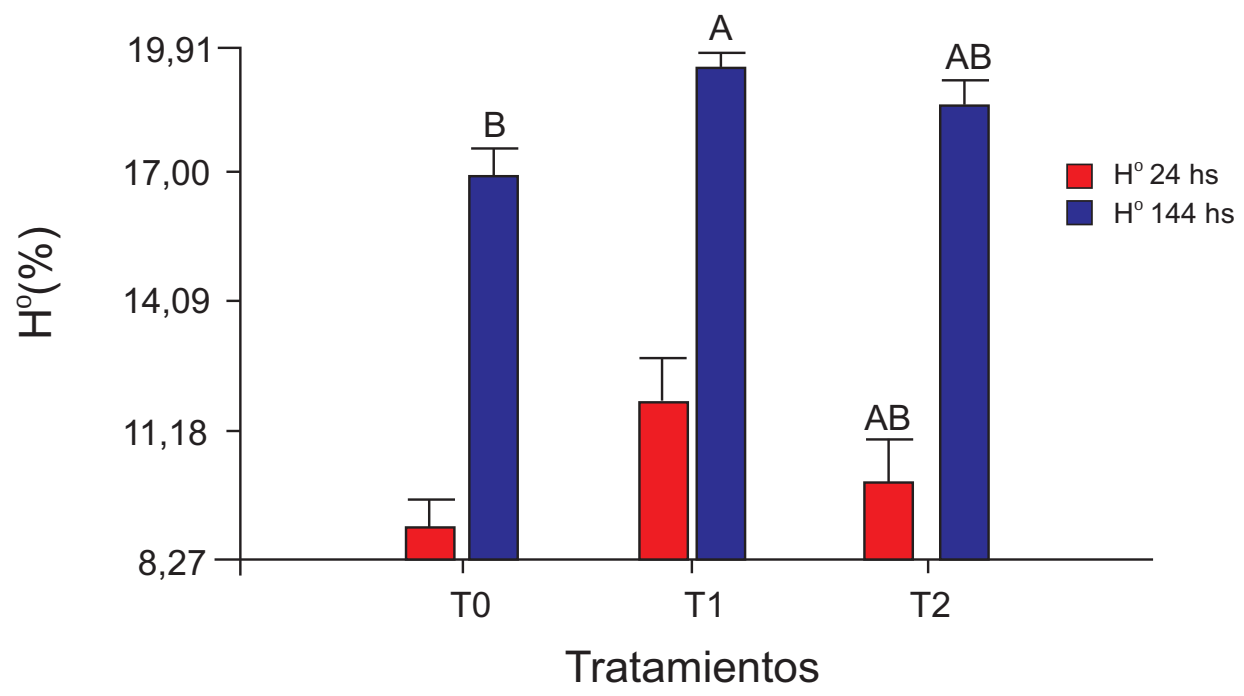

Figura 3. Contenido de agua del suelo a las 24 y 144 horas después de extraer el cultivo de lechuga tratado con diferentes dosis de enmienda de copolímero de acrilamida y acrilato de potasio. 
Finalmente, con los valores de contenido de humedad al inicio del período de sequía se establece una disminución en la cantidad de agua que se emplea en la irrigación, con respecto al que se emplearía en un suelo testigo en las mismas condiciones lo cual influye en los costos de producción. Por otra parte, en experiencias realizadas por López et al. (2013), lograron obtener mediante el uso del polímero hidrófilo a base de poliacrilamida (PAM), un incremento del $1,5 \%$ del contenido de humedad en el suelo, que representó una reducción del 12\% en el volumen de agua aplicada, por lo tanto, concluyeron que el uso del polímero hidrófilo puede ser una práctica importante para productores que deseen aplicar riegos menos frecuentes, además de reducir el tiempo de riego.

\section{CONCLUSIONES}

El cultivo de lechuga desarrollado en suelo con la enmienda del copolímero de acrilamida y acrilato de potasio presentó mayor emergencia, crecimiento, supervivencia de plantas y mejores rendimientos del cultivo. Esta enmienda aumenta el contenido de humedad del suelo lo cual se asocia con mayores rendimientos en los agroecosistemas de las regiones semiáridas y áridas.

Se sugiere que la dosis de $25 \mathrm{~kg} \cdot \mathrm{ha}^{-1}$ del copolímero de acrilamida y acrilato de potasio es suficiente para obtener buenos rendimientos del cultivo de lechuga.

Conflicto de intereses: Los autores declaran que no hay conflicto de interés.

\section{REFERENCIAS BIBLIOGRÁFICAS}

Barón, A., Barrera, I., Boada, L. \& Rodríguez, G. (2007). Evaluación de hidrogeles para aplicaciones agroforestales. Revista Ingeniería e Investigación. 27(3): 35-44.
Barreto, N. (2011). Evaluación del efecto de retenedores de agua en el establecimiento de Juniperus fláccida Schlechtendal en Ixcateopán, Gro. Chapingo México: Universidad Autónoma de Chapingo. 55p.

Brito, C., Rodrigues, F., Fernandes, M., Da Silva, L., Ricardo, N., Feitosa, J. \& Muniz, E. (2013). Síntese e caracterização de hidrogéis compósitos a partir de copolímeros acrilamida-acrilato e caulim: efeito da constituição de diferentes caulins do nordeste brasileiro. Química Nova. 36(1): 40-45.

Chatzopoulos, F., Fugit, J., Ouillon, I., Rodriguez, F. \& Taverdet, J. (2000). Étude, en fonction de différents paramètres, de l'absorption et de la désorption d'eau par un copolymère acrylamide-acrylate de sodium réticulé. European Polymer Journal, 36(1): 51-60.

Di Rienzo, J., Casanoves, F., Balzarini, M., Gonzalez, L., Tablada, M. \& Robledo, C. (2008). InfoStat, versión 2008, Grupo InfoStat, FCA. Argentina: Universidad Nacional de Córdoba.

Dranski, J., Pinto Júnior, A., Campagnolo, M., Malavasi, U., Malavasi, M. \& Guimarães, V. (2013). The effect of planting season and hydrogel on survival and initial growth of physic nut. Ciência Florestal. 23(3): 489-498.

García, A. \& Fuentes, V. (1994). Cultivos en zonas áridas: Características que mejoran sus rendimientos y uso eficaz del agua. Edición III. Murcia: Universidad de Murcia.316p.

González, C.A. (2009). Contenido de humedad del agua del suelo. Unidad 2. R.A.S.P.A. Relación Agua, Suelo, Planta, Atmósfera. Bogotá: Universidad Nacional de Colombia. 8p.

Irurtia, C., Mon, R., Holzmann, R. \& Pirolo, T. (2012). Efecto de la poliacrilamida en las propiedades físicas de un suelo arenoso. Recuperado de https://www.engormix.com/agricultura/ articulos/efecto-poliacrilamida-propiedadesfisicas-t31202.htm 
ISO 11465 (1993). Soil quality. Determination of dry matter and water content on a mass basisGravimetric method. Primera edición. Berlin: Deutsches Institut für Normung. 3p.

ISTA - International Seed Testing Association. (2014). International rules for seed testing 2014. International Seed Testing Association. Zürich, Switzerland. 5-24.

Killian, S. (2010). Introducción a la Fisiología Vegetal. Argentina: Editorial Científica Universitaria, Universidad Nacional de Catamarca. 109-165p.

Lópes, J., Da Silva, M., Saad, J. \& Angélico, T.(2010). Use of hydrogel in the survival of cuttings of eucalyptus urograndis produced with different types of substrate and water management. Ciencia Florestal. 20(2): 217-224.

López-Elías, J., Huez, M., Rueda, E., Jiménez, J., Cruz, F. \& Garrido, 0. (2013). Uso de un polímero hidrófilo en chile Anaheim (Capsicum annuum L.) bajo condiciones de invernadero. Idesia. 31(2): 77-81. doi: http://dx.doi.org/10.4067/ S0718-34292013000200011

Magalhães, A., Almeida, M., Bezerra, M. \& Feitosa, J. (2013). Superabsorbent Hydrogel Composite with Minerals Aimed at Water Sustainability. J. Braz. Chem. Soc. 24(2): 304-313. doi: http:// dx.doi.org/10.5935/0103-5053.20130039

Marques, T., Dos Santos, A. \& Marques, P. (2013). The hydrogel polymer and depth of planting in sugarcane production. IRRIGA. 18(1): 126-138. doi: http://dx.doi.org/10.15809/ irriga.2013v18n1p126

Nissen, M. \& Ovando, C. (1999). Efecto de un hidrogel humectado aplicado a las raices de Nothofagus obliqua (MIRB.) Oerst. y Nothofagm dombeyi (MIRB.) Oerst. Durante su trasplante. Agro sur. 27(2): 48-58.

Pedroza-Sandoval, A., Yáñez-Chávez, L., Sánchez-Cohen, I. \& Samaniego-Gaxiola, J. (2015). Efecto del hidrogel y vermicomposta en la producción de maíz. Revista fitotecnia mexicana. 38(4): 375381.
Trujillo, E. (2009). Plantines y retenedores de agua. Edición Especial. Revista el Semillero. 25-27. 